\section{Padrão de incorporação de fármacos antiretrovirais pelo Sistema Público de Saúde no Brasil}

\section{Patterns of antiretroviral therapy adoption by the Brazilian Public Health System}

\section{Paulo Schiavom Duarte \\ Daniel Garkauskas Ramos \\ Júlio Cesar Rodrigues Pereira \\ Departamento de Epidemiologia da Faculdade de Saúde Pública da Universidade de São Paulo.}

\section{Resumo}

Objetivo: avaliar o padrão de incorporação de fármacos antiretrovirais pelo Sistema Público de Saúde no Brasil (SUS). Métodos: baseando-se em trabalhos prévios publicados na literatura médica foram propostos três indicadores a serem utilizados na análise da incorporação de fármacos: intervalo conhecimento-incorporação, massa crítica de conhecimento e intervalo validação-incorporação. Utilizando-se as bases de dados do Departamento de Informática do SUS (Datasus) e do Sistema de Controle Logístico de Medicamentos (SICLOM) foram selecionados dois grupos de tecnologias farmacológicas (medicações antiretrovirais e medicações de dispensação em caráter excepcional). Resultados: os medicamentos antiretrovirais analisados foram incorporados de maneira mais precoce que os medicamentos de dispensação em caráter excepcional, tanto no que se refere ao intervalo "conhecimento-incorporação" quanto ao intervalo "validação-incorporação", e apresentam uma menor "massa crítica de conhecimento" para a incorporação. Conclusão: Os medicamentos antiretrovirais têm sido incorporados de maneira mais rápida, e após a publicação de um menor número de artigos científicos, quando em comparação aos medicamentos de dispensação em caráter excepcional.

Palavras-chave: Antiretrovirais. Tecnologia. Medicamentos excepcionais. Incorporação tecnológica. Indicadores. Fármacos. 


\section{Abstract}

Objective: The aim of the present scientific study is to evaluate the patterns of antiretroviral technology adoption by the Brazilian Public Health System (SUS). Methods: Based on previous articles published in scientific medical literature, three indicators to assess antiretroviral technology adoption by SUS were proposed: knowledge-adoption interval; critical mass of knowledge; and validation-adoption interval. Using the databases from the SUS Department of Information Technology (DATASUS) and from the Brazilian Logistic Center for Medication Control (SICLOM), two pharmaceutical groups were selected (antiretroviral medications and a group of high cost medications). Results: Antiretroviral medications were adopted faster than the high cost medication group when assessed on the basis of "knowledge-adoption" interval and "validation-adoption" interval. Yet, they require a lower "critical mass of knowledge" before adoption. Conclusion: Antiretroviral medications have been adopted faster and based on a lower number of scientific medical articles than a selected group of high cost medications.

Keywords: Antiretroviral. Technology. Exceptional drugs. Technology adoption. Indicators. Pharmaceuticals.

\section{Introdução}

No Brasil, o Sistema Único de Saúde (SUS) tem como uma das suas obrigações fazer valer o artigo 196 da Constituição Federal de 1988, que define: A saúdeédireito de todos e dever do Estado, garantido mediante políticas sociais e econômicas que visem à redução do risco de doença e de outros agravos e ao acesso universal e igualitário às ações e serviços para sua promoção, proteção e recuperação. Além disso, a Lei Orgânica da Saúde (Lei $\mathrm{n}^{\circ} 8.080 / 90$ ), promulgada em 1990, determina que uma das atribuições do SUS é o incremento do desenvolvimento científico e tecnológico na esfera de atuação do sistema. Desta maneira, a incorporação e a difusão de inovações decorrentes do desenvolvimento científico e tecnológico que tenham impacto na melhora do estado de saúde da população é, a princípio, uma das obrigações do sistema de saúde pública no Brasil.

No entanto, a incorporação e difusão da inovação na área de saúde é um processo complexo que envolve uma série de etapas a serem transpostas, que vão desde a validação da tecnologia para ser utilizada na prática clínica até a definição pelo gestor de saúde de prioridades a serem seguidas no processo de incorporação.

No Brasil, a produção de inovação na área de saúde tem ficado restrita a um número limitado de itens, tais como algumas vacinas, fármacos, hemoderivados e equipamentos médico-hospitalares, sendo que grande parte das inovações incorporadas ao sistema de saúde, principalmente aquelas com maior conteúdo tecnológico, tem sua origem em grandes conglomerados industriais com sede em países desenvolvidos ${ }^{1,2}$. Esta origem externa da inovação, que acaba encarecendo a sua utilização, associada a recursos limitados a serem gastos em saúde, dificultam e retardam a incorporação da inovação em muitas situações, com potenciais consequências negativas para a saúde da população.

Em razão destas etapas acima citadas, o processo de incorporação de inovação no 
sistema público de saúde pode ser bastante errático, com algumas tecnologias sendo incorporadas de maneira bastante lenta, enquanto outras são incorporadas mais rapidamente. Desta forma, é importante que se mapeie constantemente o padrão de incorporação de inovação no sistema de saúde, a fim de que se conheçam os padrões destas incorporações, bem como as possíveis especificidades existentes para cada tipo de tecnologia.

Devido à importância do programa nacional de combate à AIDS (programa nacional de DST e AIDS), criado em 1986 e atualmente denominado Departamento de DST, Aids e Hepatites Virais, existe grande influência de vários setores da sociedade no sentido de incorporação das mais novas tecnologias farmacológicas nesta área. Essa política de acesso ao tratamento antiretroviral tem como referência a Lei no $.9313 / 1996$, que estabelece a distribuição gratuita pelo SUS de medicamentos a todos os portadores de HIV/AIDS que deles necessitem (art. $1^{\circ}$ ). Ela cuida tanto da aquisição e distribuição de antiretrovirais e medicamentos para doenças oportunistas, como também estipula consensos terapêuticos, periodicamente revisados, que almejam a oferta de tratamentos seguros e eficazes ${ }^{3}$. Essa grande pressão social para o tratamento adequado e célere dos portadores de HIV/AIDS tem resultado em uma incorporação precoce dos antiretrovirais que se acredita estar associada a uma melhora na sobrevida destes pacientes $^{4}$. Desta forma, a distribuição nacional de medicamentos para portadores de HIV/ AIDS talvez possa ser considerada exemplar da incorporação célere de recursos tecnológicos, podendo ser utilizada como padrão na análise da incorporação desses recursos pelo setor público de saúde em outras patologias. No entanto, apesar da percepção de que a distribuição de antiretrovirais no Brasil é paradigmática da incorporação tecnológica eficiente, existem poucas análises quantitativas abordando esta incorporação e, aparentemente, nenhuma comparação entre essa incorporação com aquela de outras tecnologias farmacológicas.
Desta forma, o objetivo deste trabalho é avaliar quantitativamente o padrão de incorporação de fármacos antiretrovirais pelo SUS e compará-lo com o de outros fármacos.

\section{Material e Métodos}

Baseando-se em trabalhos prévios publicados na literatura médica, três indicadores foram utilizados para analisar a incorporação de antiretrovirais pelo SUS. São eles:

- Intervalo entre a $1^{\text {a }}$ publicação científica abordando a tecnologia e sua incorporação pelo sistema público de saúde (intervalo conhecimento-incorporação) - este parâmetro de análise foi sugerido em artigo prévio publicado na literatura por Booth-Clibborn ${ }^{5}$.

- Número de trabalhos abordando a tecnologia antes de sua incorporação pelo sistema público de saúde (massa crítica de conhecimento) - o conceito de massa crítica de conhecimento foi baseado em artigo científico publicado por Chaves e Albuquerque $^{6}$.

- Intervalo entre a aprovação do uso da tecnologia pelo Food and Drug Administration (FDA) nos Estados Unidos e sua incorporação pelo sistema público de saúde (intervalo validação-incorporação) - parâmetro de análise semelhante a este foi utilizado em tese de doutorado defendida por Scheffer $\mathrm{MC}^{7}$.

Identificamos dois grupos de tecnologia a serem utilizados na análise (Tabela 1): medicamentos para o tratamento da AIDS (antiretrovirais) e medicamentos de dispensação em caráter excepcional - a lista dos medicamentos antiretrovirais, bem como os dados sobre a data da sua incorporação no SUS, foram obtidas no Sistema de Controle Logístico de Medicamentos (SICLOM) (Tabela 1) - o SICLOM, que é um sistema de apoio administrativo ao Departamento de DST, Aids e Hepatites Virais, que entrou em uso em 1996 e a partir de 1997 tem registro da dispensação de diversos medicamentos antiretrovirais. 
Além dos antiretrovirais, alguns medicamentos da lista de dispensação em caráter excepcional foram selecionados para análise comparativa com os antiretrovirais. Os medicamentos excepcionais foram selecionadas por apresentarem algumas características semelhantes às dos antiretrovirais: custo elevado ${ }^{8}$, tratam patologias graves (Tabela 1), algumas das quais com mortalidade e morbidade expressivas ${ }^{9}$, seu uso está associado a melhora nos padrões de morbimortalidade das doenças ${ }^{10} \mathrm{e}$, assim como na AIDS, o gestor público sofre pressão de setores organizados da sociedade para sua incorporação $0^{8}$ - o Programa de Medicamentos de Dispensação Excepcional, que atualmente é denominado de Componente Especializado da Assistência Farmacêutica, foi iniciado em $1982^{11}$, sendo responsável por disponibilizar medicamentos para o tratamento de doenças específicas, que atingem um número limitado de pacientes, os quais na maioria das vezes os utilizam por períodos prolongados. Algumas das condições de utilização destes medicamentos englobam: Doença de Gaucher, Doença de Parkinson, Alzheimer, Hepatites B e C, pacientes renais crônicos, transplantados, portadores de asma grave, anemia, dentre outras - a lista de medicações de dispensação em caráter excepcional foi obtida da página da internet do ministério da saúde, http://portal.saude. gov.br/portal/arquivos/pdf/lista_medicamentos_excepcionais. pdf.

Os dados sobre a incorporação dos medicamentos de dispensação excepcional, bem como sua utilização ao longo dos anos, foram obtidos no DATASUS - os registros do SIA/SUS (Sistema de Informações Ambulatoriais do SUS), que estão disponíveis para consulta pública através da internet pelo Departamento de Informática do SUS (Datasus - http://www.datasus.gov.br), foram implantados em 1994.

As informações sobre a primeira publicação científica e sobre o número de artigos publicados antes da incorporação foram obtidas na base de dados do Medline (PubMed).

\section{Seleção das tecnologias em cada grupo}

A seleção das tecnologias a serem analisadas em cada um dos dois grupos (antiretrovirais e medicamentos de dispensação em caráter excepcional) baseou-se na data de incorporação pelo SUS; desta forma, utilizaram-se somente tecnologias incorporadas um ano após o início do registro das bases de dados (DATASUS e SICLOM), a fim de diminuir a chance da tecnologia já ter sido incorporada antes do registro. Logo, tecnologias que constavam do primeiro ano de registro não foram utilizadas. $\mathrm{Na}$ base de dados do SICLOM foram utilizadas tecnologias com registro inicial em $1997 \mathrm{ou}$ após (a base inicia o registro em 1996), o que resultou em 13 fármacos neste grupo de análise. Para a base de dados do DATASUS (medicamentos de dispensação em caráter excepcional e métodos diagnósticos) foram utilizadas tecnologias com registro inicial em 1995 ou após (a base inicia o registro em 1994), o que resultou em 88 medicamentos de dispensação em caráter excepcional.

Os medicamentos de dispensação excepcional foram posteriormente restritos àqueles cujo primeiro trabalho científico foi publicado após 1984. Isto reduziu o número de medicamentos utilizados neste grupo de 88 para 30 (Tabela 1). Esta restrição foi feita a fim de deixar o grupo de medicamentos excepcionais mais similar àquele dos medicamentos antiretrovirais - neste último grupo, o trabalho mais antigo registrado foi em 1985.

\section{Análises estatísticas}

Para definirmos as características gerais dos dois grupos (antiretrovirais e medicamentos de dispensação em caráter excepcional) foram calculadas as medianas dos 3 indicadores (intervalo conhecimento-incorporação, massa crítica de conhecimento e intervalo validação incorporação). O teste não paramétrico de Mann-Whitney foi utilizado para avaliar a existência de diferenças estatisticamente significativas entre os dois grupos. 
Tabela 1 - Lista de medicamentos analisados e resultados dos parâmetros para cada fármaco Table 1 - List of drugs analyzed and results of the parameters for each pharmaceutical

\begin{tabular}{|c|c|c|c|c|c|c|c|}
\hline TECNOLOGIA & CLASSIFICAÇÃO & FDA & $1^{\circ}$ TRAB. & $1 \circ \mathrm{SUS}$ & $\begin{array}{l}\text { Massa } \\
\text { Crítica }\end{array}$ & $\begin{array}{c}\text { Validação- } \\
\text { Incorporação }\end{array}$ & $\begin{array}{l}\text { Conhecimento- } \\
\text { Incorporação }\end{array}$ \\
\hline Abacavir & ANTIRETROVIRAL & 1998 & 1997 & 2001 & 215 & 3 & 4 \\
\hline Amprenavir & ANTIRETROVIRAL & 1999 & 1995 & 2001 & 183 & 2 & 6 \\
\hline Atazanavir & ANTIRETROVIRAL & 2003 & 2000 & 2004 & 9 & 1 & 4 \\
\hline Delavirdine & ANTIRETROVIRAL & 1997 & 1993 & 1999 & 127 & 2 & 6 \\
\hline Efavirenz & ANTIRETROVIRAL & 1998 & 1995 & 1999 & 116 & 1 & 4 \\
\hline Enfuvirtide & ANTIRETROVIRAL & 2003 & 1998 & 2005 & 206 & 2 & 7 \\
\hline Ganciclovir & ANTIRETROVIRAL & 1989 & 1985 & 2001 & 1060 & 12 & 16 \\
\hline Lapinavir+Ritonavir & ANTIRETROVIRAL & 2000 & 2000 & 2002 & 7 & 2 & 2 \\
\hline Nelfinavir & ANTIRETROVIRAL & 1997 & 1995 & 1998 & 94 & 1 & 3 \\
\hline Stavudine & ANTIRETROVIRAL & 1994 & 1987 & 1997 & 177 & 3 & 10 \\
\hline Tenofovir & ANTIRETROVIRAL & 2001 & 1995 & 2004 & 94 & 3 & 9 \\
\hline Thalidomide & ANTIRETROVIRAL & 1998 & 1989 & 2001 & 219 & 3 & 12 \\
\hline Zidovudine+Lamivudine & ANTIRETROVIRAL & 1997 & 1996 & 1998 & 5 & 1 & 2 \\
\hline Acetato de Lanreotida & EXCEPCIONAL & 2007 & 1989 & 2003 & 316 & -4 & 14 \\
\hline Atorvastatina & EXCEPCIONAL & 1996 & 1990 & 2002 & 544 & 6 & 12 \\
\hline Cabergolina & EXCEPCIONAL & 1996 & 1985 & 2002 & 281 & 6 & 17 \\
\hline Cloridrato de Sevelamer & EXCEPCIONAL & 1998 & 1997 & 2002 & 27 & 4 & 5 \\
\hline Donepezil & EXCEPCIONAL & 1996 & 1992 & 2002 & 432 & 6 & 10 \\
\hline Dornase alfa & EXCEPCIONAL & 1993 & 1993 & 1997 & 73 & 4 & 4 \\
\hline Entacapone & EXCEPCIONAL & 1999 & 1991 & 2002 & 151 & 3 & 11 \\
\hline Fluvastatina & EXCEPCIONAL & 1993 & 1990 & 2003 & 617 & 10 & 13 \\
\hline Imiglucerase & EXCEPCIONAL & 1994 & 1995 & 1999 & 23 & 5 & 4 \\
\hline Infliximab & EXCEPCIONAL & 1998 & 1994 & 2002 & 362 & 4 & 8 \\
\hline Interferon alfa & EXCEPCIONAL & 1986 & 1985 & 1997 & 2682 & 11 & 12 \\
\hline Interferon alfa peguilado & EXCEPCIONAL & 2001 & 1996 & 2002 & 82 & 1 & 6 \\
\hline Lamotrigina & EXCEPCIONAL & 1994 & 1985 & 1997 & 342 & 3 & 12 \\
\hline Leflunomide & EXCEPCIONAL & 1998 & 1985 & 2002 & 446 & 4 & 17 \\
\hline Micofen Mofetil & EXCEPCIONAL & 1995 & 1989 & 1998 & 338 & 3 & 9 \\
\hline Olanzapina & EXCEPCIONAL & 1996 & 1992 & 1999 & 242 & 3 & 7 \\
\hline Pramipexol & EXCEPCIONAL & 1997 & 1987 & 2002 & 171 & 5 & 15 \\
\hline Pravastatina & EXCEPCIONAL & 1991 & 1986 & 2002 & 1560 & 11 & 16 \\
\hline Quetiapina & EXCEPCIONAL & 1997 & 1991 & 2002 & 347 & 5 & 11 \\
\hline Riluzol & EXCEPCIONAL & 1995 & 1985 & 2002 & 329 & 7 & 17 \\
\hline Risperidona & EXCEPCIONAL & 1993 & 1988 & 1997 & 503 & 4 & 9 \\
\hline Rivastigmina & EXCEPCIONAL & 2000 & 1991 & 2002 & 166 & 2 & 11 \\
\hline Sinvastatina & EXCEPCIONAL & 1991 & 1986 & 2002 & 1899 & 11 & 16 \\
\hline Tacrolimus & EXCEPCIONAL & 1994 & 1987 & 1999 & 3743 & 5 & 12 \\
\hline Tolcapone & EXCEPCIONAL & 1998 & 1990 & 2002 & 213 & 4 & 12 \\
\hline Topiramato & EXCEPCIONAL & 1996 & 1987 & 2002 & 451 & 6 & 15 \\
\hline Xinafoato de Salmeterol & EXCEPCIONAL & 1994 & 1988 & 2003 & 781 & 9 & 15 \\
\hline Ziprasidona & EXCEPCIONAL & 2001 & 1993 & 2002 & 147 & 1 & 9 \\
\hline
\end{tabular}

Os parâmetros analisados são: o ano de aprovação pelo FDA (FDA), a data da 1a publicação científica (1 TRAB.), a data da incorporação no sistema público (10 SUS), número de publicações antes da incorporação ao sistema público (massa crítica de conhecimento), a diferença em anos entre a incorporação no sistema público e a aprovação pelo FDA (intervalo validação-incorporação) e a diferença em anos entre a incorporação no sistema público e a $1{ }^{\text {a }}$ publicação científica (intervalo conhecimento-incorporação).

Parameters analyzed were: the year of FDA approval (FDA), date of publication of the $1^{\text {st }}$ scientific paper (1० TRAB.), date of technology adoption by the public system (10 SUS), number of scientific articles before the technology adoption (massa critica de conhecimento), the time interval between FDA approval and the adoption of the technology (intervalo validação-incorporação) and the time interval between the $1^{\text {st }}$ scientific article and technology adoption (intervalo conhecimentoincorporação). 


\section{Resultados}

Da análise dos resultados apresentados na Tabela 2 observou-se que, utilizando os indicadores de incorporação de inovação propostos, a incorporação do uso de antiretrovirais ocorre antes daquela dos medicamentos da lista de dispensação em caráter excepcional. Observa-se que os antiretrovirais apresentam intervalo de tempo menor entre a $1^{\text {a }}$ publicação científica e a sua incorporação. A mediana deste tempo é de 6 anos para os Antiretrovirais e de 12 anos para os medicamentos de dispensação em caráter excepcional $(\mathrm{p}=0,002)$. O intervalo entre a aprovação pelo FDA e a incorporação também é menor para os antiretrovirais do que para os medicamentos de dispensação excepcional. Enquanto a mediana deste intervalo é de 2 anos para os antiretrovirais, ela é de 4,5 anos para os medicamentos de dispensação em caráter excepcional $(\mathrm{P}=0,001)$. Além disso, o número de artigos científicos publicados antes da incorporação é menor para os antiretrovirais, medianas de $127 \mathrm{e}$ de 340 artigos, respectivamente $(p=0,004)$.

\section{Discussão}

Os resultados obtidos são coerentes com a percepção existente atualmente de que a incorporação dos antiretrovirais ocorre de maneira precoce quando em comparação a outras tecnologias na área de saúde. No capítulo 11 dos "Indicadores de Ciência, Tecnologia e Inovação" publicado pela FAPESP em 2004 (http://www.fapesp.br/indicadores2004/volume1/ cap11_vol1.pdf), os autores relatam a importância do programa de controle da infecção pelo HIV/AIDS e se referem a este como paradigma em termos de eficiência e modelo de intervenção em saúde pública. Desta forma, os resultados apresentados corroboram a celeridade na incorporação dos antiretrovirais pelo SUS, e fornecem uma análise estatística inferencial àquele conhecimento qualitativo sobre a incorporação de fármacos no sistema público de saúde.

Vale ressaltar, nessa discussão, a dificuldade existente na obtenção de indicadores sobre incorporação de tecnologia em saúde. Apesar deste tema estar cada vez mais presente na literatura científica médica ${ }^{12,13}$, são raros os artigos que o abordam valendo-se de indicadores ${ }^{5}$; desta forma, as análises realizadas nos artigos sobre incorporação de tecnologia em saúde baseiam-se mais em avaliações qualitativas sobre esta incorporação do que em avaliações quantitativas. Em decorrência desta escassez de indicadores bem estabelecidos, dos três indicadores utilizados nas análises realizadas neste artigo, um deles foi "emprestado" da cienciometria ${ }^{6}$ e o outro utilizado em tese de doutorado? Logo, um trabalho que ainda necessita ser realizado no campo da "Avaliação de Tecnologia em Saúde" é o desenvolvimento de indicadores que possam ser utilizados na análise quantitativa de incorporação de tecnologias.

No que tange especificamente ao indicador "massa crítica de conhecimento", é importante referir que a interpretação deste é complexa, visto que a incorporação de tecnologias após a publicação de poucos

Tabela 2 - Resultados para os 3 indicadores propostos para os dois grupos de fármacos

Table 2 - Results for the 3 proposed indicators for the two pharmaceutical groups

\begin{tabular}{lccc}
\hline & $\begin{array}{c}\text { Massa Crítica de } \\
\text { Conhecimento }\end{array}$ & $\begin{array}{c}\text { Intervalo Validação- } \\
\text { Incorporação }\end{array}$ & $\begin{array}{c}\text { Intervalo Conhecimento- } \\
\text { Incorporação }\end{array}$ \\
\hline Mediana AntiRetroviral & 127 & 2,00 & 6,00 \\
Mediana Excepcional & 340 & 4,50 & 12,00 \\
Mann-Whitney & $\mathrm{p}=0,004$ & $\mathrm{p}=0,002$ & $\mathrm{p}=0,001$ \\
\hline
\end{tabular}

Resultados das medianas dos indicadores e do teste de Mann-Whitney (p) para comparação dos dois grupos de fármacos (antiretrovirais e medicações de dispensação em caráter excepcional).

Results of the median values for the indicators and Mann-Whitney test ( $p$ ) values for the comparisons between the two pharmaceutical groups (antiretrovirals and high cost medications). 
artigos científicos pode ser interpretada tanto como algo positivo, e que reflete a atenção do gestor à literatura científica, com consequente incorporação célere daquelas tecnologias cujas evidencias comprovam sua utilidade clínica, quanto como algo negativo, em que tecnologias são incorporadas sem um embasamento científico muito grande. Além disso, o número de artigos publicados também não expressa a qualidade destes artigos, nem o seu conteúdo. Um refinamento neste indicador que poderia melhorar sua qualidade e que pode ser desenvolvido para análises futuras é a incorporação do fator do impacto da revista em que o artigo foi publicado. Desta forma, não mediríamos somente o número de artigos publicados, mas sim a soma dos fatores de impacto das revistas em que estes foram publicados. No entanto, independente das limitações referidas e dos potenciais mecanismos para refinamento deste indicador, este pode ser bastante útil para demonstrar diferenças nos padrões de incorporação de diferentes tecnologias, mas sem fazer "juízo de valor" sobre qual padrão de incorporação é realmente o mais adequado, mesmo porque o padrão "ideal" pode variar de tecnologia para tecnologia e na dependência da patologia em que a tecnologia é empregada.

\section{Conclusão}

Feitas as considerações acima, os resultados do nosso trabalho permitem concluir que os medicamentos antiretrovirais são incorporados de maneira mais precoce e embasados em um menor número de trabalhos científicos quando comparados com os medicamentos de dispensação em caráter excepcional.

\section{Referências}

1. Gadelha CA, Quental C, Fialho BC. [Health and innovation: a systemic approach in health industries]. Cad Saude Publica 2003; 19(1): 47-59.

2. Gadelha CA. [Development, health-industrial complex and industrial policy]. Rev Saude Publica 2006;40: Spec 11-23.

3. de Azevedo Meiners CM. [Pharmaceutical patents and public health: challenges for the Brazilian antiretroviral treatment policy]. Cad Saude Publica 2008 24(7):146778.

4. Okie S. Fighting HIV--lessons from Brazil. N Engl J Med 2006 11; 354(19): 1977-81.

5. Booth-Clibborn N, Packer C, Stevens A. Health technology diffusion rates. Statins, coronary stents, and MRI in England. Int J Technol Assess Health Care 2000; 16(3): 781-6.

6. Chaves CV, Albuquerque EM. Desconexão no sistema de inovação no setor saúde: uma avaliação preliminar do caso brasileiro a partir de estatísticas de patentes e artigos. Econ Aplic 2006; 10(4): 523-9.

7. Scheffer MC. Aids, tecnologia e acesso sustentável a medicamentos: a incorporação dos anti-retrovirais no Sistema Único de Saúde [tese de doutorado]. São Paulo: Departamento de Medicina Preventiva da Faculdade de Medicina da USP; 2008.
8. Vieira FS. Gasto do Ministério da Saúde com medicamentos: tendência dos programas de 2002 a 2007. Rev Saude Publica 2009; 43(4): 674-81.

9. Wong JB, McQuillan GM, McHutchison JG, Poynard T. Estimating future hepatitis C morbidity, mortality, and costs in the United States. Am J Public Health 2000 90(10): 1562-9.

10. Tuffic-Burban S, Babany G, Lonjon-Domanec I, Deltenre P, Canva-Delcambre V, Dharancy S, et al. Impact of pegylated interferon and ribavirin on morbidity and mortality in patients with chronic hepatitis $\mathrm{C}$ and normal aminotransferases in France. Hepatology 2009; 50(5): 1351-9.

11. Silva RCS. Medicamentos Excepcionais no Âmbito da Assistência Farmacêutica no Brasil [tese de doutorado]. Rio de Janeiro: Fundação Oswaldo Cruz; 2000.

12. Stevens A, Milne R, Burls A. Health technology assessment: history and demand. J Public Health Med 2003 ;25(2): 98-101.

13. Lettieri E, Masella C, Nocco U. Budgeting and health technology assessment: first evidence obtained from proposal forms used to submit the adoption of new technology. Int J Technol Assess Health Care 2008; 24(4): 502-10. 